\title{
Pityriasis lichenoides-like secondary syphilis and neurosyphilis in a HIV-infected patient
}

\author{
Maciej Pastuszczak, Wioletta Woźniak, Andrzej K. Jaworek, Anna Wojas-Pelc
}

Department of Dermatology, Jagiellonian University Medical College, Krakow, Poland Head: Prof. Anna Wojas-Pelc MD, PhD

Postep Derm Alergol 2013; XXX, 2: 127-130

DOI: $10.5114 /$ pdia.2013.34166

\begin{abstract}
Papulo- and vesiculo-necrotic lesions are rare manifestations of secondary syphilis. Until now it has been described only in HIV-infected patients with advanced stages of immunosuppression. This case report describes an unusual case of PLEVA-like syphilis in a 33-year-old man with newly diagnosed HIV infection. Despite that the CD4 cells level and viral load did not indicate the advance stage of immunosuppression, the unusual manifestation of syphilis and neurosyphilis occurred. The presented case indicates the need for HIV screening in every patient with syphilis especially when the clinical manifestation is unusual. Importance of syphilis testing in every case with atypical rashes should be also highlighted.
\end{abstract}

Key words: PLEVA-like, syphilis, HIV, neurosyphilis.

\section{Introduction}

Syphilis is a sexually transmitted infection caused by Treponema pallidum. The secondary stage is characterized by the high variability of cutaneous findings. However, unusual clinical manifestations of syphilis may be more common in HIV-infected patients [1].

\section{Case report}

A 33-year-old otherwise healthy homosexual man presented at the dermatology clinic with a 3-week history of progressive, non-pruritic rash associated with mild headache and photophobia. Two weeks prior, chicken pox was diagnosed in the patient and treatment with acyclovir was started.

The skin examination revealed numerous, randomly scattered papules of various size and plaques on the face, trunk, and extremities (Figure 1). Some of the lesions showed central necrosis (Figure 2). Generalized lymphadenopathy was present. Neurological examination revealed no abnormalities.

Blood serology for syphilis was positive (VDRL 1/128, TPHA: positive, FTA 1/32000, FTA-ABS: positive). Both ELISA and Western blot tests for HIV were positive. The CD4 cells level was 430/ $\mu$ l and HIV RNA load was 25000 copies/ml.
Negative results were obtained for tests for hepatitis B and C. Cerebrospinal fluid (CSF) examination showed the elevated white blood count (30 cells/ $\mu$ l, $100 \%$ of lymphocytes), elevated protein concentration (85 mg/dl; range to $45 \mathrm{mg} / \mathrm{dl}$ ), positive CSF-VDRL and CSF-TPHA. Syphilis, neurosyphilis and HIV infection were diagnosed. The patient was treated with crystalline penicillin intravenously at a dose of 24 million units per day, for 21 days. Skin lesions cleared completely and blood VDRL titer declined to $1 / 8$ within 6 weeks after completing the therapy. The patient was referred to the department of infectious diseases for further tests and treatment of HIV infection.

\section{Discussion}

Syphilis is a systemic illness, caused by the spirochete Treponema pallidum. The World Health Organization (WHO) estimates that there are 12 million new cases of syphilis each year, with more than $90 \%$ occurring in developing countries [2]. Based on data from 2003-2004, it is estimated that more than $60 \%$ of syphilis cases occur in men who have sex with men (MSM). A large proportion of these persons are also infected with HIV [1].

The disease has been arbitrarily divided into three stages (primary, secondary and tertiary). Signs and

Address for correspondence: Maciej Pastuszczak MD, PhD, Department of Dermatology, Jagiellonian University Medical College, 8 Skawińska St, 31-066 Krakow, Poland, phone: +48 602228 796, e-mail: mpastuszczak@wp.pl Received: 31.08.2012, accepted: 10.02.2013. 


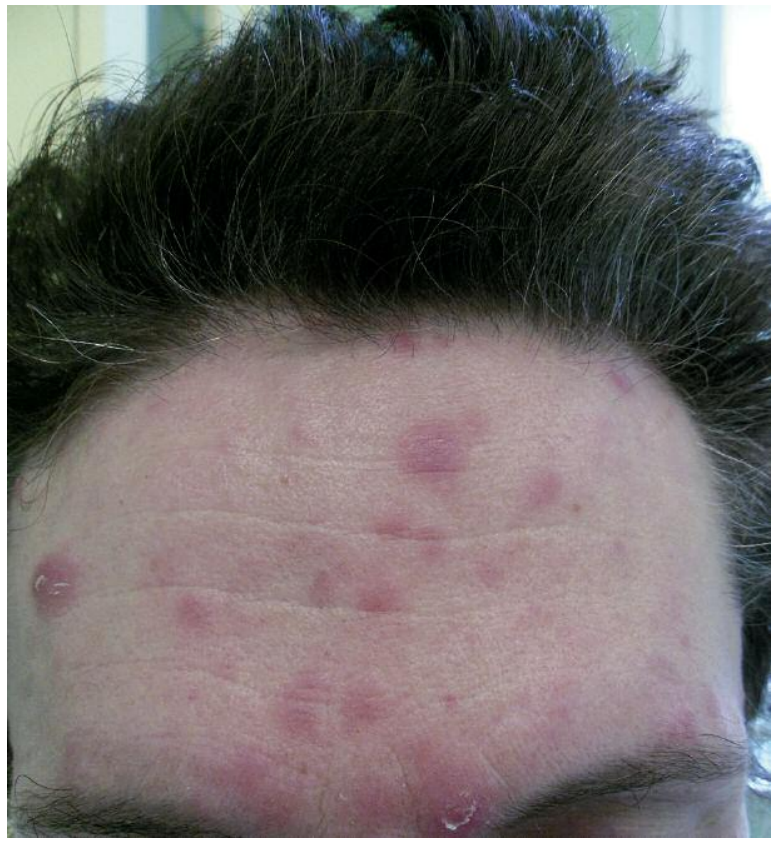

Figure 1. PLEVA-like syphilis. Papules and plaques on the forehead

symptoms of secondary syphilis typically begin 4-10 weeks after the appearance of a chancre (primary syphilis lesion). Lesions of secondary syphilis result from the hematogenous dissemination of treponemes from syphilitic chancres. A frequent first manifestation of this stage of the disease is an asymptomatic, delicate, light red macular eruption found on the flanks. Untreated, the macules disappear within 2 weeks. Subsequently, the maculopapular or papulosquamous exanthema appears affecting the hands and soles [3]. However, pustular, papular, lichenoid, nodular, ulcerative, plaque-like, annular and even urticarial and granulomatous forms can occur [4-7]. Due to a large variety of skin manifestations of secondary syphilis, the disease has to be differentiated from a number of dermatological disorders. Most common are: primary HIV, HIV immune reconstitution syndrome, pityriasis rosea, psoriasis, erythema multiforme, tinea versicolor, lichen planus, drug eruptions, viral exanthema, seborrheic dermatitis and scabies. Secondary syphilis is a systemic process so general symptoms such as fatigue, malaise, sore throat, fever, lymphadenopathy, muscular and joint pain may occur. Renal, gastrointestinal and hepatic disease is also possible [8].

Some recent studies have suggested that syphilis in HIV co-infected patients causes transient increases in the viral load and decreases in the CD4 cell count increasing the degree of immunosuppression [9]. Thus, the natural course of syphilis may be altered in HIV positive patients. HIV co-infection may be associated with multiple chancres (up to $70 \%$ of patients) which are larger and deeper than in HIV-uninfected persons. Approximately one fourth

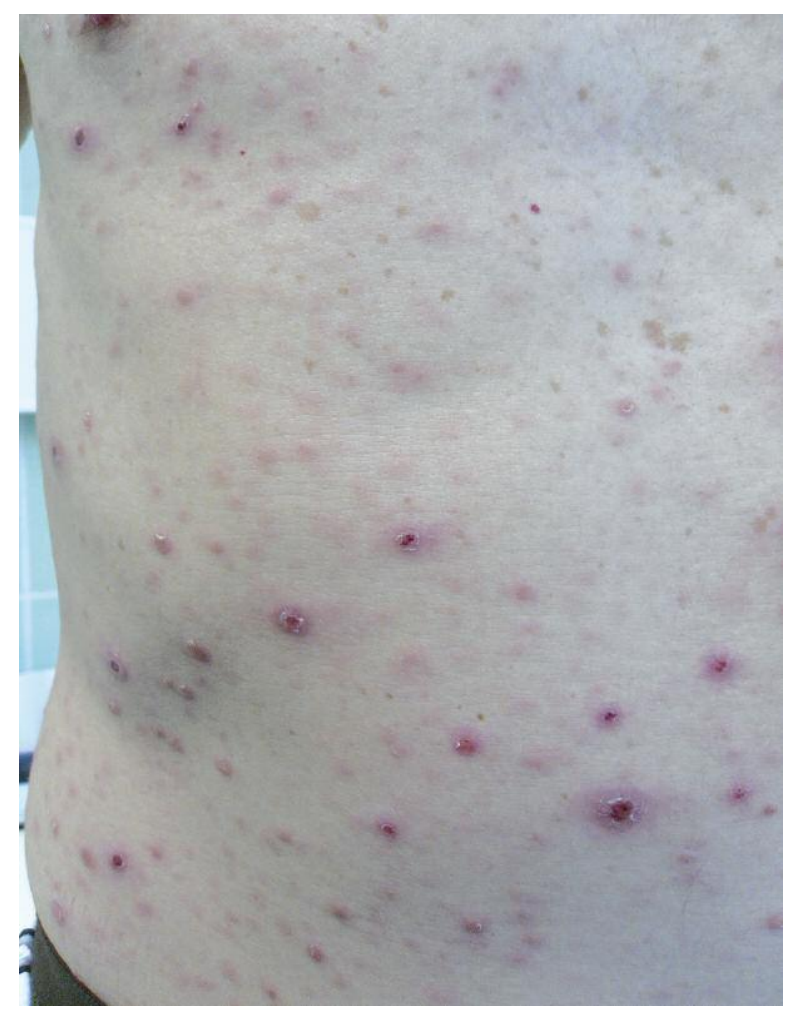

Figure 2. PLEVA-like syphilis. Scattered papules on the trunk. Some lesions with central necrosis

of HIV-infected patients present with concomitant lesions of both primary and secondary stages of syphilis at the time of diagnosis. Additionally, significantly more HIV-positive patients present with more aggressive secondary disease, which is accompanied by greater constitutional symptoms, greater organ involvement, atypical rashes, and when treated with penicillin - a predilection for developing Jarish-Herxheimer-Lukasiewicz reaction [1]. Moreover, unusual and long-lasting syphilitic lesions in HIV coinfected patients may cause a significant delay in diagnosis $[10,11]$.

In our patient we did not find the coexistence of chancre and secondary syphilis lesions. The patient was without general symptoms and Jarish-Herxheimer-Lukasiewicz reaction did not occur.

Neurosyphilis can be divided into early and late forms. Early neurosyphilis affects the CSF, cerebral blood vessels, and meninges. It occurs within weeks to a few years after primary infection, and may be asymptomatic. Symptomatic forms of early neurosyphilis include meningitis, with or without cranial nerve or eye involvement, and meningovascular disease or stroke. Late neurosyphilis affects brain or spinal cord parenchyma, and usually occurs years to decades after primary infection. Manifestations of late neurosyphilis include general paresis, a rapidly progressive dementia with psychotic features, and tabes dorsalis, a spinal cord disorder with sensory ataxia and bowel and bladder dysfunction [12]. 
There is no criterion standard for the diagnosis of neurosyphilis. In the appropriate clinical settings, CSF pleocytosis of $>5$ cells $/ \mathrm{mm}^{3}$ and elevated CSF protein concentration are suggestive of neurosyphilis. The diagnosis of neurosyphilis is more difficult in HIV-infected individuals since up to $60 \%$ of them may demonstrate CSF abnormalities including pleocytosis even without coexisting syphilis. In such cases, cutoff equal and greater than 20 cells $/ \mathrm{mm}^{3}$ may be more specific for neurosyphilis diagnosis. In the absence of visible blood contamination, a positive CSF-VDRL result is specific and establishes the diagnosis of neurosyphilis; however, the sensitivity of CSFVDRL against clinical diagnosis is only $30 \%$ to $70 \%$. In contrast, treponemal tests are sensitive, but nonspecific. A negative CSF-FTA-abs and/or CSF-TPHA test result virtually excludes the diagnosis of neurosyphilis [12].

In the presented case there was no doubt about the diagnosis of neurosyphilis. The patient had both neurological symptoms (i.e. headache and photophobia) and CSF abnormalities including positive CSF-VDRL result.

Approximately one third of patients with early syphilis have invasion of treponemes in the CSF, regardless of their HIV status. If untreated, treponemes may either spontaneously clear from CNS, persist or progress clinically (i.e. meningovascular syphilis or later form such as tabes dorsalis) [12]. Its mechanism is unclear. However, it is believed that HIV-infected individuals are less susceptible to clear treponemes from CNS. Some case reports and retrospective studies found that neurosyphilis in HIV-infected individuals may occur more frequently and progress more rapidly [13]. However, until now it has not been confirmed in prospective trials.

A recent study of Marra et al. found that persons with serum RPR (Rapid Plasma Reagin) test titers of $1: 32$ or higher and HIV-infected persons with CD4 cell counts of $350 / \mu$ or lower were at higher risk of CNS syphilis. Thus, it has been suggested, as previously discussed, that HIV associated immunodysregulation may account for the impaired clearance of treponemes from CNS [14]. In our patient the CD4 cells count was 430/ $\mu$ l. It suggested that the host defense was not significantly impaired. Despite that neurosyphilis has occurred. Thus, other than immunologic factors of treponemes CNS spontaneous clearance must be considered.

According to the International Union Against Sexually Transmitted Infection (IUSTI), duration of the treponemocidal level of antimicrobial should be at least 7-10 days to cover a number of division times (approximately $30 \mathrm{~h}$ ). Thus, the first line therapy options in early syphilis (i.e. primary, secondary and early latent) are: 1) single dose of benzathine penicillin at a dose of 2.4 million units intramuscularly (level of evidence $\mathrm{lb}$ - obtained from at least one randomized control trial) which provides a treponemocidal penicillinaemia for up to 4 weeks or 2) procaine penicillin of 600000 units intramuscularly daily for 10-14 days (level of evidence Ilb - obtained from at least other type of well-designed quasi-experimental study). In cases of penicillin allergy, alternatives are: 1) doxycycline of $200 \mathrm{mg}$ daily for 14 days, 2) tetracycline of $500 \mathrm{mg}$ four times orally daily for 14 days (both have the level of evidence III - obtained from well-designed non-experimental descriptive studies), 3) erythromycin of $500 \mathrm{mg}$ four times orally daily for 14 days (level of evidence IV - obtained from expert committee reports or opinions). Azithromycin shows good anti-treponemocidal activity in animal studies and early open studies showed that it appear effective in early syphilis [15]. However, intrinsic resistance to azithromycin has been described in some T. pallidum strains [16].

Despite controversies, current guidelines recommend that treatment of early syphilis in patients with concomitant HIV infection should be given as for non-HIVinfected patients. Some specialists recommend routine CSF-examination in HIV-positive patients [15].

In our Department, because of the increased risk of neurosyphilis, we perform lumbar puncture in all HIV-infected patients. Additionally we define the degree of immunosuppression by determining the CD4+ count and HIV viral load. All patients with clinical and/or laboratory findings consistent with neurosyphilis diagnosis are treated with neurosyphilis protocols (i.e. benzyl penicillin of 12-24 million units intravenously daily, as 3-4 million units every $4 \mathrm{~h}$ during 18-21 days) [15].

In conclusion, after the advent of HIV infection epidemic, it may be more common to see cases of syphilis with unusual presentation. Our case highlights the importance of syphilis testing in every case with atypical rashes. All patients who have syphilis should be also tested for HIV infection. We would like to emphasize that even mild and non-specific neurological symptoms such as headache and/or photophobia in a patient with syphilis may suggest neurosyphilis.

\section{References}

1. Karp G, Schlaeffer F, Jotkowitz A, et al. Syphilis and HIV coinfection. Eur J Int Med 2009; 20: 9-13.

2. Hook EW, Peeling RW. Syphilis control - a continuing challenge. N Eng J Med 2004; 35: 122-4.

3. Lautenschlager S. Cutaneous manifestations of syphilis. Am J Clin Dermatol 2006; 7: 291-304.

4. Kumar B, Gupta S, Muralidhar S. Mucocutaneous manifestations of secondary syphilis in north Indian patients: a changing scenario? J Dermatol 2001; 28: 137-44.

5. Martin DH, Mroczkowski TF. Dermatologic manifestations of sexually transmitted diseases other than HIV. Infect Dis Clin North Am 1994; 8: 533-82.

6. Carbia SG, Lagodin C, Abbruzzese M, et al. Lichenoid secondary syphilis. Int J Dermatol 1999; 38: 53-5.

7. Papini M, Bettacchi A, Guiducci A. Nodular secondary syphilis. Br J Dermatol 1998; 138: 704-5.

8. Goh BT. Syphilis in adults. Sex Transm Infect 2005; 81: 448-52.

9. Palacios R, Jimenez-Onate F, Aquilar M, et al. Impact of syphilis infection on HIV viral load and CD4 cell counts in HIV-infected patients. J Acquir Immune Defic Syndr 2007; 44: 356-9. 
10. Pastuszczak M, Snarska-Drygalska A, Wojas-Pelc A. Syphilis and HIV infection - "a dangerous combination". Dermatol Estet 2011; 13: 362 - 365.

11. Karlinska-Jachowska M, Chmielnicki P, Dziankowska-Bartkowiak B, et al. Syphilis - issue of the 21st century. Post Derm Alergol 2007; 5: 233-7.

12. Marra CM. Update on neurosyphilis. Curr Infect Dis Rep 2009; 11: $127-34$

13. Collis TK, Celum CL. The clinical manifestations and treatment of sexually transmitted diseases in human immunodeficiency virus-positive men. Clin Infect Dis 2001; 32: 611-22.

14. Marra CM, Maxwell CL, Smith SL, et al. Cerebrospinal fluid abnormalities in patients with syphilis: association with clinical and laboratory features. J Infect Dis 2004; 189: 369-76.

15. French P, Gomberg M, Janier M, et al. IUSTI: 2008 European guidelines on management of syphilis. Int J STD AIDS 2009; 20: 300-9.

16. Lukehart SA, Godornes C, Molini B, et al. Marcolide resistence in Treponema pallidum in the United States and Ireland. N Engl J Med 2004; 351: 154-8. 